\title{
ESTUDIO SOBRE LOS MEDIOS DE COMUNICACIÓN DE ECUADOR PARA LA DIFUSIÓN DE CIENCIA, TECNOLOGÍA E INNOVACIÓN EN EL NUEVO ESCENARIO CONSTITUCIONAL
}

\author{
STUDY OF THE MEDIA OF ECUADOR FOR THE DISSEMINATION OF SCIENCE, \\ TECHNOLOGY AND INNOVATION IN THE NEW CONSTITUTIONAL SCENARIO
}

\section{GUILLERMO DEL CAMPO ${ }^{1}$, MAO GARZÓN², ALBERTO FRANCO ${ }^{3}$, YAMIL LAMBERT4 ${ }^{4}$, WELLINGTON VILLOTA ${ }^{5}$}

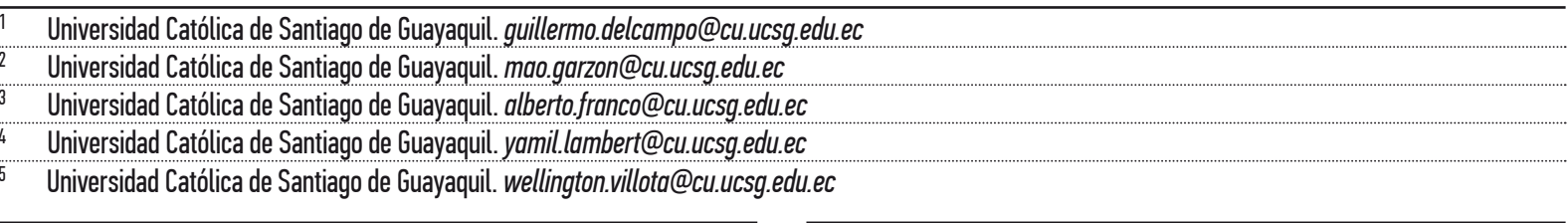

RESUMEN

El nuevo marco constitucional establecido en Ecuador a partir del 2008 que dio paso a las reformas de la Ley Orgánica de Educación Superior aprobada en el 2010 y la nueva Ley de Comunicación que entró en funcionamiento en el 2013 modifica el actual escenario sobre el funcionamiento de los medios de comunicación, divide el espectro para su operatividad y articula nuevas propuestas y modos de producción. El presente informe contextualiza el nuevo escenario en el que transcurren los medios de comunicación públicos, privados y comunitarios y su integración con la universidad para la difusión de ciencia, tecnología e innovación y su impacto en la comunidad en general. En una sociedad en donde la ciencia y la tecnología se hacen presentes de múltiples maneras, la búsqueda de una cultura científica es indispensable para la existencia de una verdadera democracia participativa en el que los medios de comunicación se constituyen en unos mediadores sociales de primer orden entre ciudadanos e instituciones. Los resultados evidencian que la difusión, transmisión y recepción de información siguen siendo escasos y de bajo impacto a pesar del nuevo escenario legal que actualmente rige en el país; de igual forma, se analizó la inversión pública y privada en educación, ciencia, tecnología e innovación como uno de los factores que predominan en el impacto que éstas áreas puedan tener en la sociedad. Se demuestra la importancia entre el incremento de la inversión en $C+\mid$ y la masificación de la información para desarrollar una cultura científica acorde a los nuevos escenarios.

PALABRAS CLAVE: ciencia, tecnología, medios de comunicación, innovación, ley de comunicación.
The new constitutional framework established in Ecuador from 2008 which led to the reform of the Organic Law on Higher Education adopted in 2010 and the new Communications Act which came into force in 2013 modifies the current scenario on the functioning of the media, divides the spectrum for their operation and articulates new ideas and modes of production. This report contextualizes the new scenario in which pass the public, private and community media and its integration with the university for the dissemination of science, technology and innovation and its impact on the community. In a society where science and technology are present in many ways, the search for a scientific culture is essential for the existence of genuine participatory democracy in which the media constitute a prime social mediators between citizens and institutions. The results show that the diffusion, transmitting and receiving information remain scarce and low impact despite the new legal scenario currently prevailing in the country; Likewise, public and private investment in education, science, technology and innovation as one of the factors that dominate the impact that these areas can have on society is analyzed. The importance between increased investment in $C \& I$ and the spread of information to develop a scientific culture according to new scenarios is shown.

KEYWORDS: science, technology, media, innovation, media law. 
INTRODUCCIÓN

Los medios en el Ecuador se encuentran regulados en su funcionamiento y ámbito de desarrollo por un marco legal determinado fundamentalmente por la nueva Ley Orgánica de Comunicación y confluyen con el mismo objeto las regulaciones contenidas en el reglamento a dicha ley, así como las disposiciones existentes en la Ley Orgánica de Educación Superior y finalmente las que norman el ámbito de acción en procesos de elección existentes en el código de la democracia (Asamblea Legislativa Nacional, 2009).

La Ley Orgánica de Comunicación aprobada por la Asamblea Nacional en el 2013, establece en el Art. 8 la Prevalencia de Contenidos y dice "Los medios de comunicación en forma general difundirán contenidos de carácter informativo, educativo y cultural en forma prevalente. Estos contenidos deberán propender a la calidad y ser difusores de los valores y los derechos fundamentales consagrados en la constitución y en los instrumentos internacionales de derechos humanos" (Ley Orgánica de Comunicación, 2013).

Desde el ámbito legal, existe la obligación para la difusión de educación y cultura a través de los medios de comunicación en Ecuador, los mismos que están inmersos en las definiciones sobre todo lo relacionado a ciencia tecnología e innovación. Esto no implica que obligatoriamente los medios tengan que dedicarse exclusivamente a la difusión de este tipo de contenidos. Sin embargo la obligatoriedad se da parcialmente cuando en el Art. 74 numeral 3, de la misma Ley de Comunicación expresa que se debe "destinar una hora diaria, no acumulable para programas oficiales de tele-educación, cultura, salubridad y derechos elaborados por los ministerios o Secretarias con competencia en estas materias", (Ley Orgánica de Comunicación, 2013).

En ese contexto, se vuelve necesario definir los conceptos de medios de comunicación, su articulación y papel que juegan en el nuevo marco legal, para tal efecto, el artículo 5 de la Ley Orgánica de Comunicación, 2013, expresa que "para efectos de esta ley se consideran medios de comunicación social a las empresas, organizaciones públicas, privadas y comunitarias, así como a las personas concesionarias de frecuencias de radio y televisión, que prestan servicio público de comunicación masiva, que usan como herramientas medios impresos o servicios de radio, televisión y audio y video por suscripción, cuyos contenidos pueden ser generados o replicados por el medio de comunicación a través de internet, (Ley Orgánica de Comunicación, 2013, p.5)".

Este nuevo marco legal introduce cambios en la gestión de comunicación, modificando las estructuras que se han creado a partir de las nuevas tecnologías, como la cultura DIY (Do it yourself), que aporta la profusa teoría planteada por (Jenkins, 2003), que concentra sus recientes investigaciones en la manera como los individuos aprovechan y combinan fuentes mediáticas de la cultura contemporánea y sugiere que la convergencia mediática debe ser entendida como un proceso cultural en vez de un punto final tecnológico.

Jenkins (2009), cree que la convergencia no es el simple encuentro tecnológico en el que confluyen los diferentes medios, sino que es un proceso cultural propio de los seres, aboga por una interpretación de la convergencia que no tenga en cuenta solamente el aspecto tecnológico, material, sino toda la red compleja de interacciones entre los sistemas técnico, industrial, cultural y social. Al distanciarse del determinismo tecnológico, se otorga una gran importancia al proceso de recepción de las audiencias mediáticas, afirmándose que en realidad la convergencia no tiene lugar mediante aparatos mediáticos, por muy sofisticados que éstos puedan llegar a ser, sino que se produce en el cerebro de los propios individuos, así como mediante sus interacciones sociales con otros.

Al destacar este componente simbólico, humano, de la convergencia, Márquez (como se citó en Jenkins, 2009) subraya que ésta es tanto un proceso corporativo de arriba abajo como un proceso de abajo arriba dirigido por los consumidores; de esa forma, la convergencia corporativa, mediática, coexiste con la convergencia popular, humana, dentro de un sistema híbrido cuyos elementos se influyen y retroalimentan mutuamente, (Márquez, 2010).

Al converger los diferentes medios, estos se convierten en el vehículo por el cual el conocimiento fluye. Los medios por sí solos únicamente hacen conocer la existencia de una nueva situación o el nacimiento de una nueva teoría, la investigación profunda de esa teoría no se hace a través de todos y cada uno de los medios, será privilegio de aquellos que no se vean limitados por cuestiones de espacio o condicionalidad, normalmente infieren en este espacio los medios especializados, revistas, webs, entre otros. Su presencia hace conocer la 
existencia del conocimiento, de la ciencia o del descubrimiento.

Hay que considerar que los "mass media" se presentan en la actualidad de varias formas, puesto que ya no se está restringido a la presentación tradicional, más aún cuando el desarrollo de la tecnología ha hecho que incursionen en el mundo de la difusión de las diferentes condiciones de la realidad cualquier forma de expresión individual que se presta para ser leída o recibida por muchos o por pocos, dependiendo de que el hecho se convierta en una "necesidad colectiva" a partir de las tendencias que marcan los lectores como de "interés social".

En ocasiones esta tendencia es marcada por los gobiernos dependiendo de necesidades que se originan por ejemplo en situaciones de guerra "Un fuerte impulso para ello, como se lo encuentra en la necesidad que hubo de formar en EE.UU. a miles de militares para su supervivencia y eficacia militar en la II Guerra Mundial, lo que hizo que se crearan y distribuyeran miles de películas y otros materiales de enseñanza." (Pardo, 2009).

Conforme se indica, existe en el presupuesto legal la obligatoriedad de que los medios, especialmente los audiovisuales, otorguen parte de sus tiempos aire en beneficio de la difusión colectiva de contenidos culturales, pero en ellos no existe la obligatoria necesidad de difusión educativa, estableciendo una profunda diferencia entre medios culturales, medios educativos y medios comerciales y privados.

"Los contenidos culturales y educativos en los medios de comunicación de Ecuador no representan ni el $10 \%$, una cifra que no significa un saldo rojo, pero sí es "pobre" (Superintendencia de Comunicación , 2014).

El informe analizó a 31 de los 78 medios de comunicación que operan en Ecuador, considerados de mayor sintonía y lectoría a nivel nacional y regional, muestra que los contenidos educativos en la televisión representan el 5\%, en la radio el 4\% y en los medios impresos el 9\%, (Superintendencia de Comunicación, 2014).

Esto contrasta con los contenidos de entretenimiento que son los que más copan los medios de comunicación; en el caso de la televisión llegan al $59 \%$. La cifra en radio es del $30 \%$ mientras que el porcentaje más bajo se registra en la prensa escrita $(9 \%)$.

El estudio muestra que los contenidos informativos en televisión llegan al $20 \%$, y en radio y prensa al 42 y $33 \%$, respectivamente.

Estos resultados marcan una relación directa sobre el número de medios de comunicación que existen en el país y su orientación comercial, 99 canales que emiten su señal tanto a nivel nacional como regional son privados o comunitarios con orientación comercial y de lucro, de las 1205 emisoras existentes en el país, el $97 \%$ pertenecen al sector privado desde 1950 y de ellos 256 emisoras pertenecen a grupos empresariales que acumulan emisoras con fines comerciales; mientras que existen a nivel nacional 12 medios impresos, todos orientados a actividades privadas comerciales (Jordán, 2010).

A pesar de la división del espectro radioeléctrico que contempla la nueva ley de comunicación: $34 \%$ para comunitarios, $33 \%$ para privados y $33 \%$ para públicos, surgen dudas sobre los mecanismos de llevar a la práctica ésta normativa, no solo por la complejidad para equilibrar el funcionamiento de otros modelos de medios de comunicación desde las bases formativas a ciudadanos con nuevos roles como actores y participantes de los cambios sociales, así como desde la academia la generación de investigación para el desarrollo de nuevos paradigmas en la construcción y difusión de nuevos contenidos, la difusión de la ciencia y tecnología es escasa en la medida que la propuesta en investigación y desarrollo en el país sigue la misma línea.

En cuanto a la generación de política en la Educación Superior los medios no generan en si un contenido orientador, porque fundamentalmente los medios en el país tienen una orientación hacia lo comercial, siendo entonces los campos educativos una especie de sectores tabú, porque no son generadores de publicidad, lo que los deja al margen de cualquier opinión, especialmente en lo que respecta a la educación superior.

La nueva ley de medios promueve la creación de medios públicos y comunitarios en instituciones educativas tanto públicas como privadas, un ejemplo de eso son los canales de televisión de las universidades Católica de Cuenca, Católica de Guayaquil y la Escuela Superior Politécnica del Litoral, quienes se encuentran inmersas en el desarrollo de sistemas de televisión que vayan a apoyar la difusión educativa, sin embargo las estructuras de desarrollo de carreras de tercer nivel se encuentran en fases de estudio y etapa inicial. En la Universidad Católica de Santiago de Guayaquil, se emprendió desde abril de 2015 la creación de un modelo de educación a distancia en convergencia de medios para tercer y cuarto nivel, estudio que estará culminado en dos años con experimentación y diseño de cómo hacer y producir espacios educativos interactivos en el marco de la tecnología digital. 
La creación de nuevos medios y la entrada de la digitalización en la transmisión tanto en radio como en televisión, así como el impulso desde plataformas tecnológicas de medios impresos, adquiere mayor relevancia en el país, al considerarse a la tecnología como elemento transversal en el desarrollo de nuevos mecanismos de difusión de contenidos, tanto desde quien crea los mensajes como de quien los recibe, lo que conlleva un replanteamiento en el paradigma de la educación tradicional.

\section{DESARROLLO}

Ecuador está en proceso para poner en marcha un plan nacionalquedesarrolle las capacidades y potencialidades en investigación y desarrollo tecnológico, que desde la perspectiva de las políticas de C\&T, resultan necesarios para apuntalar la economía del conocimiento. Estas capacidades están relacionadas con el número necesario de investigadores ubicados en centros, laboratorios, equipos y proyectos en al menos las siguientes áreas o sectores: recursos genéticos y biodiversidad; biotecnología aplicada a salud humana y animal; biotecnología agrícola; bio-remediación; nanotecnología; nuevos materiales, biomateriales y biopolímeros; informática y desarrollo de software; nuevas fuentes de energía; conflicto, democracia y tecnologías sociales. Todo lo cual debe estar suficientemente respaldado por políticas públicas que permitan al país contar con programas de formación, retención, repatriación y promoción de los talentos humanos nacionales.

En efecto es claro la necesidad de fortalecer activos fundamentales para el desarrollo del país, su productividad y competitividad tales como la innovación, medida por el número de patentes, la presencia y calidad de Centros de Desarrollo Tecnológicos y el nivel tecnológico de las empresas y el capital humano, que propone alcanzar metas al 2017 de al menos dos mil investigadores activos y acreditados (Secretaria Nacional de Educación Superior, Ciencia y Tecnología, 2013). Para obtener esto es indispensable una fuerte disciplina fiscal tanto en el gobierno nacional cuanto en los gobiernos locales y en las instituciones de educación superior en lo relacionado a la inversión en el sector de ciencia y tecnología. Adicionalmente es importante complementar estrategias orientadas a atraer financiamiento externo para fortalecer las capacidades científicas y tecnológicas endógenas, incentivar la generación del conocimiento protegible, y crear el marco institucional que estimule al sector privado a tener una mayor inversión en $\mathrm{C}+\mathrm{T}+\mathrm{I}$.

Es indispensable fortalecer el Sistema Nacional de Ciencia, Tecnología e Innovación, respetando la autonomía universitaria para garantizar la creación científica, en un ambiente de libertad, condición necesaria para lograr la creatividad propia de toda la empresa científica. En efecto es vital preguntarse ¿Qué políticas nacionales se han implementado? ¿Qué leyes incentivan la formación de investigadores o a las empresas innovadoras?, ¿Cuáles son las orientaciones para el rediseño de instituciones, especialmente de las que deben especializarse en la creación de conocimiento? En todo esto ¿Cuál es el rol asignado a las universidades públicas? En ese sentido los señalamientos de la nueva constitución y el plan nacional del buen vivir presentan elementos que pueden ser vistos por parte de las universidades como una oportunidad para la creación de tecnología, difusión y transferencia de tecnología, investigación y desarrollo tecnológico y, desde luego, como el núcleo generador de procesos innovadores que involucren al sector empresarial interesado en la comercialización de tecnología.

Es primordial las iniciativas en lo relativo a ciencia, tecnología e innovación involucrar a las universidades, tanto en el nivel de pregrado como de posgrado, mediante el desarrollo de programas y proyectos de I\&D que a través de sus resultados tengan un impacto sobre la calidad y accesibilidad de la mayor parte de la población a la educación, salud, medio ambiente, agricultura, biotecnología médica, farmacéutica, redes de cómputo y telecomunicaciones.

Ecuador pronuncia sus demandas de ciencia y tecnología en el plan nacional de desarrollo en el cual están trazadas las políticas propuestas por el Gobierno en materia de C\&T para el futuro inmediato. Aquí es posible encontrar lineamientos para la producción de conocimientos y por lo tanto las pautas y las orientaciones de la investigación universitaria. Por lo tanto la formación de doctores, investigadores, la publicación de trabajos de investigación universitaria, la adecuación de los perfiles profesionales, deben tener en cuenta las políticas de ciencia y tecnología, además de los lineamientos que 
constan en el plan nacional de desarrollo, pero también los acelerados cambios que se están generando en las fronteras del conocimiento a nivel internacional.

Las acciones de ciencia y tecnología están desde 2008 en el Ecuador estrechamente vinculadas a las de Educación Superior, y no solamente por la preponderancia de la producción científico-tecnológica de las IES que, tal como se ha indicado en el pasado tienden a percibir como realidad latinoamericana. Bajo ese contexto, bien se podría señalar que antes del actual gobierno no existían políticas coordinadas de desarrollo de C\&T, ni marcos legales que impulsen un cambio en la matriz productiva, tampoco un cambio en el modelo de enseñanza en todos los niveles, lo que ha permitido actualmente que la Educación Superior gane una relevancia inusitada, al menos para lo que eran los usos y costumbres en el Ecuador, y su destino ha quedado emparentado con el del desarrollo de la investigación científica y tecnológica en un nuevo marco de políticas públicas que la pone en el centro de la escena.
Desde el 2008 a la actualidad, se puede observar un incremento en la inversión de recursos humanos, a través de los programas de becas de formación en el exterior y el programa Prometeo; en infraestructura, con planes de inversión en Universidades Escuelas politécnicas, junto a la construcción de cuatro nuevas universidades, en investigación, con financiamiento a proyectos y programas de investigación, entre los más importantes.

Sin embargo uno de los aspectos que entran a debate es el objetivo de recuperar el rol director del Estado en la educación superior, tal como lo promulga la (Asamblea Nacional Constituyente, 2008) a través del Consejo Nacional de Evaluación Académica (CONEA, 2009).

De igual forma, se crea el Consejo de Evaluación Académica y Aseguramiento de la Calidad de la Educación Superior, el mismo que se puso marcha y entró en funcionamiento con el objetivo de recuperar la calidad académica de tercer nivel y cuarto nivel en el país (CEAACES, 2013).

La (Secretaría de Educación Superior, Ciencia y Tecnología, 2007) proponía un documento

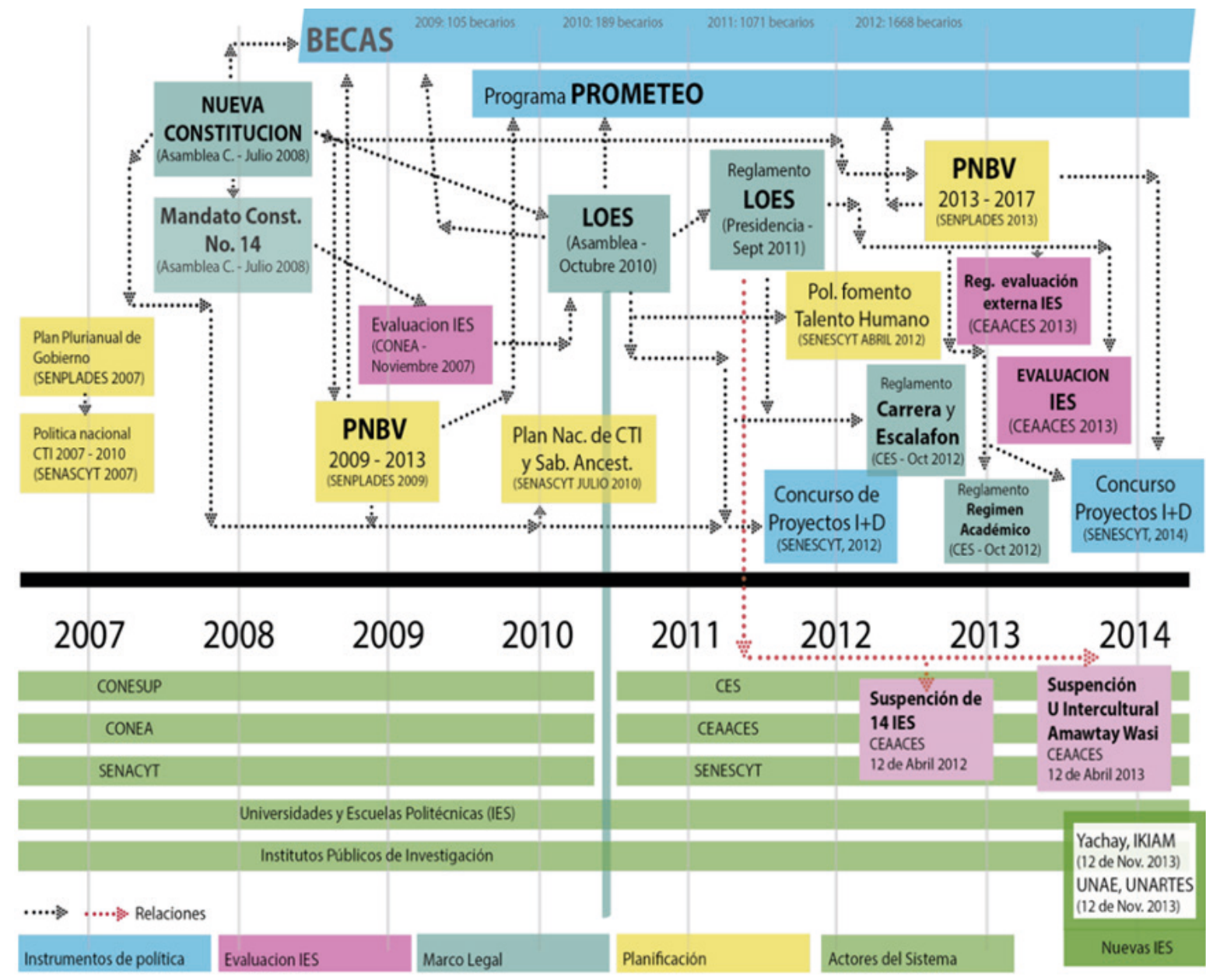

Figura 1. Modelo estratégico de inversión en educación, ciencia y tecnología en el Ecuador, período 2007-2014. Fuente: Senescyt (2014). 
de planificación específica para el sector de la educación, con el objetivo de plantear la creación de áreas estratégicas y establecía como insumo para el desarrollo humano, la ciencia y la tecnología, junto a un plan nacional de desarrollo que se planteaba como quinta meta 'impulsar la investigación, la ciencia y la tecnología' (Secretaría Nacional de Planificación y Desarrollo, 2007).

Sin embargo, no sería hasta que entró en vigencia la nueva constitución que, finalmente, se constituiría un nuevo espacio para el desarrollo de políticas públicas con injerencia sobre las actividades de investigación científica y tecnológica. La constitución sentó las bases y, dos años después, la (Ley Orgánica de Educación Superior, 2011), junto a su posterior reglamentación, un año más tarde, hizo operativos algunos de sus principales principios.

No se trata entonces, de enviar cámaras y transmitir hechos noticiosos relacionados a ciencia y tecnología, debido a que el problema no está únicamente en el medio o mensaje, sino principalmente en el receptor, a sabiendas de conocer el real impacto, interés, motivación y uso de la información para su real utilidad.

El modelo de transmisión de contenido impuesto por los medios desde lo privado, debe migrar hacia la apuesta que deben existir a través de lo público y comunitario. No se trata de condenar las actividades de medios privados, sino estar conscientes que durante 50 años se construyó un paradigma sobre modos de recepción de mensajes y modelos de contenidos, por lo tanto el rol que juegan las instituciones educativas en los tres niveles como laboratorios de experimentación, debe permitir la formación de escenarios más apropiados para generar mayores respuestas con base a las necesidades actuales de la sociedad que se está construyendo.

Históricamente en Ecuador solo han coexistido medios privados, regulados hasta el 2013 por una Ley de Comunicación que fue reformada en 1975, lo que permitió a muchos grupos de poder político y económico controlar los medios de comunicación, una realidad que no solo le compete a Ecuador, sino a toda Latinoamérica, como lo señala Beltrán (2005) que manifiesta la concentración de la propiedad de los medios de comunicación masiva en pocas manos se ha acentuado en Latinoamérica en elevada proporción tanto en lo transnacional como en lo nacional.

En efecto, (Instituto de Prensa y Sociedad, 2004), halló un alto grado de concentración en la estructura de las industrias culturales y de telecomunicación. Verificó que en cada mercado nacional las cuatro firmas principales dominan, en promedio, algo más del $60 \%$ del público y de la facturación. Y puso en evidencia que en varios de los países los grupos económicos más poderosos controlan las empresas de comunicación y que ello conduce a la formación de conglomerados de múltiples medios.

"Los gobiernos de la región han venido favoreciendo crecientemente la inversión privada, inclusive en los sectores de la comunicación usualmente tenidos por estratégicos, como la televisión, la radio y las telecomunicaciones; y en vez de propiciar la instauración de políticas públicas democratizantes de la comunicación $\mathrm{y}$, como tales, potenciadoras de la participación del pueblo en el proceso de comunicación, no pocos de dichos regímenes están inclusive impulsando la ultraliberal desregulación -preferentemente por medio de decretos, no por leyes- que beneficia a los sectores, internos y externos, que consideran que la mejor política de comunicación es, si posible, ninguna... o aquellas que, reformuladas a conveniencia de sus intereses, contribuyan a mantener intacta su hegemonía en nombre del "libre flujo de la información”. (Beltrán, L., 2005, p15).

En el Ecuador de similar manera a lo que ha ocurrido en la mayoría de los países de la región, los medios masivos, especialmente la televisión comercial, han puesto en marcha la toma de posiciones empresariales para el desarrollo de las más modernas y sofisticadas tecnologías de comunicación que han hecho posible la uniformización de los modelos empresariales, la estandarización de la producción de contenidos y la mercantilización ampliada del proceso comunicativo.

En este contexto, resulta oportuno meditar sobre la importancia de introducirnos en el conocimiento del poder así como de los intereses que están detrás de cada uno de los medios masivos, ya que prácticamente ningún canal, radio o prensa actúa de forma independiente, la mayoría, o casi la totalidad de ellos, están relacionados a una gran empresa influyente de la comunicación que es la que, por cierto, le permite su supervivencia económica.

Para el efecto de este estudio, y dado que la programación regular en los medios, tanto de televisión como de radio, ha sido concentrada hacia la divulgación de noticias generales, entretenimiento y deportes, se analizó la parrilla de programación de dos canales de televisión, que por su papel y rol se creyó conveniente 
exponerlos, tal es el caso del canal comercial Ecuavisa, líder del rating de sintonía (Ibope, 2014); y Ecuador TV, canal 7, medio público que emite su señal a nivel nacional y está operando desde el 2007; además es importante señalar los esfuerzos que están desarrollando canales comunitarios como UCSG TV, único medio universitario que emite su señal a nivel nacional e internacional que pertenece a una institución de educación superior, por invertir en sistemas y proyectos de investigación que determinen una metodología de producción de contenidos en la difusión y emisión de mensajes bajo el nuevo formato digital que entrará en vigencia en el 2018.

Con 45 años de emisión, la cadena privada Ecuavisa, fortalece su sistema de información en la producción de noticias nacionales e internacionales, además hace énfasis en el desarrollo de programas de salud, con frecuencia semanal y diaria respectivamente, orientados a la prevención de enfermedades y soluciones inmediatas en el tema de salud, no cuenta en su parrilla de programación con programas que permitan divulgar en un escenario propio ciencia y tecnología e innovación. Aquello está supeditado a pequeñas cápsulas informativas dentro de los noticieros respectivos para informar sobre algún avance y que la noticia en contexto tenga relevancia. A esta propuesta se agrega la obligatoriedad de transmitir media hora diaria un proyecto llamado Educa, desarrollado por el Ministerio de Educación, cuyo propósito es abordar diferentes temas de cultura y ciencia con el objetivos de coadyuvar a la educación informal de niños y jóvenes en el país.

De igual forma, retransmite un programa de la Secretaría Nacional de Comunicación (SECOM), cuyo objetivo es la búsqueda de la identidad cultural, la diversidad y el rescate de valores y costumbres de los pueblos, así como el reconocimiento de un estado pluricultural a través de la exposición de piezas audiovisuales.

La programación se complementa con franjas de ocio y entretenimiento, una lógica que se repite en todos los medios televisivos privados y comerciales del país.

El canal del Estado empezó a funcionar en abril del 2007, y el contenido que transmite es de productores independientes nacionales y extranjeros, así como convenios para retransmitir programación de canales públicos de varios países. Al ser un canal nuevo, los contenidos de Ecuador TV aún no despiertan un interés por parte de la comunidad, su programación es variada y los cambios permanentes no definen sus objetivos.

Los programas que se emiten son culturales y no se evidencia fomento a la difusión de ciencia, tecnología e innovación de lo que se está generando en el país, ni a través de redes de información paralelas o programas puntuales que permitan la masificación de información de CTI.

Aunque el canal público tenga reconocimiento por parte de la ciudadanía, un estudio elaborado por (CIESPAL, 2009), determinó que existe un gran porcentaje de ciudadanos que no ven el canal y quienes nunca lo ven constituyen la tercera parte en cada ciudad donde se desarrolló la encuesta.

\section{CONCLUSIONES}

La llamada alfabetización científica, apoyada en buena medida por los medios de comunicación como modo de alcanzar la cultura científica en la sociedad, ha sido cuestionada desde hace algunos años, pues implica utilizar el denominado "modelo de déficit" refutado por algunos de los investigadores del ámbito de la percepción pública, para quienes es muy impreciso determinar lo que la gente debe saber para considerarse alfabetizado científicamente, Sin embargo, sin comunicación social de la ciencia es imposible alcanzar cultura científica.

La necesidad de acrecentar esta cultura científica en la sociedad se vincula en los días actuales a la gobernanza. En el caso de la ciencia, este concepto aparece relacionado a la toma de decisiones sobre la orientación pública de la actividad científica, basada en la deliberación.

En una sociedad en donde la ciencia y la tecnología se hacen presentes de múltiples maneras, la búsqueda de una cultura científica es indispensable para la existencia de una verdadera democracia participativa. Y en una verdadera democracia, los medios de comunicación se constituyen en unos mediadores sociales de primer orden entre los ciudadanos y las instituciones, en una sociedad donde está presente la ciencia y la tecnología, y en donde la opinión y la participación de los ciudadanos en asuntos públicos es mandato constitucional, la información que permita el desarrollo de una cultura científica se hace indispensable.

Es imposible pensar en una difusión con impacto de $\mathrm{C}+\mathrm{T}+\mathrm{I}$ sino se revisan procedimientos de enseñanza-aprendizaje desde las escuelas, en un cambio cultural que paulatinamente en Ecuador se empieza a fomentar y en donde los medios de comunicación en general, bajo 
un nuevo marco regulatorio, no practiquen y hagan énfasis en la función social que les compete. Los medios no reemplazan los centros educativos, pero si pueden articular propuestas innovadoras y establecer mecanismos estratégicos con los centros de educación superior que permita a la ciudadanía gozar de mayores y mejores beneficios para adquirir conocimientos complementarios, solo allí serán verdaderos protagonistas del desarrollo del país.

Si los medios ofertan, lo que la sociedad demanda, es responsabilidad de quienes forman sociedades de elevar el nivel de demanda, por lo que una división del espectro radioeléctrico sino viene acompañado de políticas y acciones a través de una situación sinérgica entre Estado, universidad, empresa privada y sociedad, por si misma no logrará un cambio en el paradigma actual.

\section{REFERENCIAS BIBLIOGRÁFICAS}

Andes, A. d. (12 de Agosto de 2014). Agencia de Noticias Andes. Obtenido de Agencia de Noticias Andes: http://www.andes.info. ec/es/noticias/supercom-alerta-sobre-pococontenido-educativo-medios-comunicacionecuador.html

Asamblea Legislativa Nacional. (27 de Abril de 2009). Código de la Democracia. Codigo de la Democracia. Quito, Pichincha, Ecuador: Suplemento del R.O. 578.

Asamblea Legislativa Nacional. (12 de Octubre de 2010). Ley Organica de Educación Superior. Ley Organica de Educación Superior. Quito, Pichincha, Ecuador: Suplemento del R.O. 298.

Asamblea Legislativa Nacional. (22 de julio de 2013). Ley Organica de Comunicacion. Ley Organica de Comunicacion. Quito, Pichincha, Ecuador: R.O. 022.

Asamblea Nacional Constituyente. (2008). Ley Orgánica de Eduación Superior. Quito: Asamblea Nacional.

Beltrán, L. (12 de04 de 2005). La comunicación para el desarrollo en latinoamérica: un recuento de medio siglo. Obtenido de http://www. infoamerica.org: http://www.infoamerica. org/teoria_textos/lrb_com_desarrollo.pdf

CEAACES. (2013). Suspendidas por falta de calidad, el cierre de catorce universidades en Ecuador, Quito. Informe de la suspensión de universidades

CIESPAL. (2009). Percepción sobre los medios públicos en Ecuador. Quito: CIESPAL.
CONEA. (2009). Evaluación de desempeño institucional de las Universidades y Escuelas Politécnicas del Ecuador. CONEA, Quito.

Consejo de Regulación de la Información y de la Comunicación, CORDICOM. (19 de Diciembre de 2013). Reglamento a la Ley Orgánica de Comunicación. Quito, Pichincha, Ecuador: R.O. 147.

Ibope. (2014). Medición de rating de sintonía de canales de televisión. Quito: Ibope.

Instituto de Prensa y Sociedad. (2004). Estudio sobre la concentración de la propiedad de los medios en América Central . México: IPS-OEA.

Jenkins, H. (2003). transmedia storytelling en Technology Review. Revista del Massachusetts Institute of Technology (MIT), 4.

Jenkins, H. (2009). Confronting the Challenges of Participatory Culture . Massachusetts, EE.UU: Massachusetts Institute of Technology.

Jordán, R. (2010). Los medios de comunicación en Ecuador. Quito,: Universidad Los Hemisferios.

Ley Orgánica de Comunicación. (25 de Junio de 2013). Ley Orgánica de Comunicación de Ecuador. Ley Orgánica de Comunicación de Ecuador. Quito, Pichincha, Ecuador: Asamblea Nacional.

Ley Orgánica de Educación Superior. (2011). Estatutos orgánicos para procesos del Consejo de Educación Superior. Quito: LOES.

Márquez, I. (2010). Artículo: Reseña de "Convergence Culture. La cultura de la convergencia de los medios de comunicación" de Jenkins, Henry. CIC. Cuadernos de Información y Comunicación, 319-338.

Pardo. (2009). EL FIN DE LOS MEDIOS MASIVOS. BUENOS AIRES: La Crujia Ediciones.

Secretaría de Educación Superior, Ciencia y Tecnología. (2007). Planificación estratégica para el sector de la Educación Superior. Quito: SENESCYT.

Secretaria Nacional de Educación Superior, Ciencia y Tecnología. (2013). Plan Nacional de investigación 2013-2017. Quito: Senescyt.

Secretaría Nacional de Planificación y Desarrollo. (2007). Plan Estratégico Nacional 2007-2013. Quito: SENPLADES.

Superintendencia de Comunicación . (2014). Informe sobre contenidos educativos en los medios de comunicación de Ecuador. Quito: Superintendencia de Comunicación (SUPERCOM). 